\title{
Spatial Variations of Galaxy Number Counts in the Sloan Digital Sky Survey. II. Test of Galactic Extinction in High Extinction Regions
}

\author{
Naoki Yasuda, Masataka Fukugita \\ Institute for Cosmic Ray Research, University of Tokyo, Kashiwa, 277-8582, Japan \\ yasuda@icrr.u-tokyo.ac.jp \\ and \\ Donald P. Schneider \\ Department of Astronomy and Astrophysics, 525 Davey Laboratory, Pennsylvania State \\ University, University Park, PA 16802
}

\begin{abstract}
Galactic extinction is tested using galaxy number counts at low Galactic latitude obtained from five band photometry of the Sloan Digital Sky Survey. The spatial variation of galaxy number counts for low extinction regions of $E(B-V)<$ 0.15 is consistent with the all-sky reddening map of Schlegel, Finkbeiner, \& Davis (1998) and the standard extinction law. For higher extinction regions of $E(B-$ $V)>0.15$, however, the map of Schlegel et al. (1998) overestimates the reddening by a factor up to 1.4, which is likely ascribed to the departure from proportionality of reddening to infrared emissivity of dust. This result is consistent with the analysis of Arce \& Goodman (1999a) for the Taurus dark cloud complex.
\end{abstract}

Subject headings: dust, extinction — techniques: photometric

\section{Introduction}

The Sloan Digital Sky Survey (SDSS: York et al. 2000) provides an unprecedented widefield imaging survey of the sky with homogeneous, few-percent-error photometry. It allows study of the spatial variation of the projected galaxy number density over the wide field. In our previous publication (Fukugita et al. 2004, Paper I), it was shown that the variation of the galaxy number counts is accounted for by the Galactic extinction and large-scale galaxy clustering. 
Galaxy counts provide a clean test of the Galactic extinction integrated out to the edge of the Milky Way, provided that the galaxies are sampled to sufficiently faint magnitude and their numbers are sufficiently large (see, e.g., Hubble 1934; Burstein \& Heiles 1982). The test does not need any models or external calibrations to derive extinction. Moreover, using SDSS photometric data, extinctions in five-bands can be determined independently and the extinction curve can be derived. The primary limitation of this technique is that one cannot explore the small scales where spatial variations of the galaxy distribution are strongly affected by galaxy clustering. The mean amount of Galactic extinction can be well documented, and the error for scale larger than a degree can be made smaller than the order that concerns us. The study in Paper I indeed verified the validity of the reddening map of Schlegel et al. (1998, SFD) on the scale of a degree and the extinction laws of Cardelli et al. (1989) and O'Donnell (1994) to within 5\% for five optical colours. More recently, a similar but more detailed analysis reveals in very low extinction regions slight underestimates of extinction in the SFD map, which are likely due to the contamination of infrared emission from galaxies in the map of dust far infrared emission (Yahata et al. 2006).

Our study of extinction in Paper I was limited to regions of low extinction $[E(B-V)<$ 0.15 ) since the galaxies used are derived from the main survey area of SDSS (Abazajian et al. 2003, DR1) that intentionally avoids the region of large extinction to explore extragalactic science. A significant amount of SDSS imaging data, however, has been obtained at low Galactic latitudes for the purpose of photometric commissioning and calibrations. A part of such surveys, that include the Orion region, are published in Finkbeiner et al. (2004). The purpose of the present paper is to extend our study to high extinction regions and examine the validity of the reddening map and the extinction law given by SFD.

We note that some indication was already reported that the SFD reddening map may not be correct for high extinction regions from a study of stars in the background of the Taurus dark cloud complex (Arce \& Goodman 1999a). Arce \& Goodman suggested that extinction derived from SFD is overestimated by a factor of $1.3-1.5$ in regions with $A_{V}>0.5 \mathrm{mag}$, or $E(B-V)>0.16$. Our study will test this result in the Orion region.

In Section 2 we describe the sample, data selection and the procedure to derive extinction from galaxy number counts. Section 3 presents the result together with some discussion. A summary is given in Section 4. 


\section{Data and analysis}

The imaging by SDSS telescope (Gunn et al. 1998, 2006) is carried out with the SDSS ugriz filters (Fukugita et al. 1996). Our analysis is based on the data given by Finkbeiner et al. (2004). We use the data of stripe 82 (southern equatorial stripe) which are derived from photometric scans, runs $211(82 \mathrm{~S}), 259(82 \mathrm{~N})$, and $273(82 \mathrm{~S})$. This is the only stripe in Finkbeiner et al. (2004) for which a north-south pair of stripes, which are needed to form a contiguous area, are observed. The region covers $11.55<\alpha<91.55$ and $-1.25<\delta<+1.25$, corresponding to $125^{\circ} \lesssim l \lesssim 207^{\circ}$ and $-62^{\circ} \lesssim b \lesssim-10^{\circ}$. Figure 1 shows the region covered by current data set overlaid in the reddening map of SFD for the Galactic southern hemisphere. The total area of our data set, $200 \mathrm{deg}^{2}$, is about $10 \%$ of that covered by Paper I derived from SDSS DR1. The mean selective reddening $E(B-V)$ increases from 0.03 at a high Galactic latitude to $\approx 1$ in the Orion region close to the Galactic plane, as seen in Figure 2 , which shows $E(B-V)$ of SFD averaged over the $2.5^{\circ}$ square regions along the stripe. We note that extinction in dark cloud regions is patchy, and $E(B-V)$ occasionally reaches $>10$ mag for small regions, typically for $15^{\prime}$.

An object catalogue and five colour band images are given in Finkbeiner et al. (2004). The catalogue is created by photometric pipeline photo v5_4_25, which is used to produce Data Release 2 (Abazaiian et al. 2004) and later data releases (Abazajian et al. 2005; Adelman-McCarthy et al. 2006, 2007). We select galaxies primarily using objType parameter in the catalogue, but drop objects which are flagged as EDGE, BRIGHT, SATURATED, or BLENDED. It sometimes happens that some objects are not detected in all five bands. We judge the detection using logical OR of BINNED1, BINNED2, and BINNED4 flag set per band, which mean that objects are detected in a $1 \times 1,2 \times 2$, and $4 \times 4$ binned image, respectively. The entries that are not detected in a given band are not included in our number counts in that band. There are some entries in the catalogue whose corresponding objects appear to be much too faint for the quoted magnitudes; their surface brightness is indeed faint. The occurrence of this type of spurious detection is common in all colour bands. When the surface brightness distribution is plotted for the galaxy sample, these objects produce a spike at nearly zero surface brightness. Since the reason for the inclusion of such entries in the catalogue is not clear, we apply a surface brightness cut at the level of sky background to exclude those entries in the $r$ band. This procedure automatically removes the surface brightness spikes in the $g, i$ and $z$ bands. For the $u$ band, there are many true galaxies that have low surface brightness, so we cannot separate the fake spike from the tail of true galaxies; we simply assume that the removal of the spike in the $r$ band also removes spurious objects in the $u$ band. This procedure rejects about $2 \%$ of the $r$ band selected galaxies in the dereddened magnitude range of $17.5<r<19.5$. We find that most of these entries are flagged as nopetro, but if we would use nopetro as the selection criterion we would miss an 
additional $3 \%$ of objects, which are mostly true galaxies.

To account for patchy extinction structure in a heavily reddened region, we divided the total area of 200 square degrees into pixels of $2.3^{\prime} \times 2.3^{\prime}$. This pixel size was adopted to match that of the SFD reddening map $\left(2.372^{\prime}\right)$. There are 135,200 such pixels in our area. All pixels are sorted in the order of increasing $E(B-V)$ values of the SFD map and grouped every 4225 pixels, so that the sum of the area of each group amounts to $\left(2.5^{\circ}\right)^{2}$, which is large enough to determine extinction from galaxy number counts. Figure 3 shows the mean $E(B-V)$ value of each group. Half of the groups have $E(B-V)<0.1$. Since the group of the largest $E(B-V)$ spans too wide a range of reddening, we subdivide it into four: namely the last four points have been grouped with 1056 pixels (one quarter of 4225). The last dataset includes pixels with $E(B-V)$ from 1 to 33; it is dropped from our analysis.

The present analysis differs from that in Paper I in that contiguous regions of 2.5 degree squares are considered. Such an analysis is not appropriate to deal with the sample that includes high extinction regions, since extinction is very patchy for dark cloud regions and the average of extinction over a large area does not necessarily match the magnitude offset calculated from galaxy count:1 1 . For this reason we divide the area into small regions and assemble the region according to extinction values in the present paper. For low extinction regions, the details of the binning are not important.

We use the Petrosian magnitude (see Stoughton et al. 2002; Strauss et al. 2002) in our study. Petrosian magnitudes are suitable for the analysis given in this paper, since the Petrosian radii are unaffected by the foreground extinction and Petrosian magnitudes measure the same fraction of the flux of galaxies regardless of their foreground extinction. This means that we can correct the extinction properly just by subtracting the extinction value in each band. This does not hold for other galaxy magnitudes such as isophotal or aperture magnitudes. We have to note that Petrosian magnitudes are not corrected for seeing variations unlike model magnitudes which are PSF-convolved model fits. The fraction of total magnitude measured by Petrosian magnitude will change as galaxies become smaller as a result of worse seeing (Blanton et al. 2001). This effect is only a few percent for the galaxies used in this study $\left(17.5<r<22.0\right.$ or $\left.1.0^{\prime \prime}<r_{50}<1.3^{\prime \prime}\right)$ and does not affect our results. We refer astrometric calibrations to Pier et al. (2003) and photometric calibrations of the main survey to Smith et al. (2002) (see also Hogg et al. 2001; Tucker et al. 2006) and Ivezić et al. (2004). Since the secondary standard star "patches" are sparse or nonexistent for much of the Orion

\footnotetext{
${ }^{1}$ For example, in an area where half of the region is clear and the other half is extincted by 20 magnitudes, then the mean extinction would be $10 \mathrm{mag}$. However, galaxies can be observed in the clear area and the number of galaxy will be half that expected for the case without extinction. This leads us to infer that the magnitude offset would be about $0.5 \mathrm{mag}$.
} 
region, photometric calibrations for the current data use the übercalibration algorithm (See Finkbeiner et al. 2004; Gunn et al. 2006, for details).

We derive the mean extinction-free galaxy number count (differential count) $\bar{N}(m)$ from the entire sample in low extinction regions of $E(B-V)<0.1$ by employing the SFD reddening map and the default standard extinction law, $k(r)=A_{r} / E(B-V)=2.751$ (Table 6 of SFD). We take this mean relation as the reference. We then count the number of galaxies for each group without applying the extinction correction, and fit to the reference count by shifting the amount of magnitude $\Delta m$, i.e., as $\bar{N}(m+\Delta m)$; this $\Delta m$ represents the extinction in the specific region.

We first work with the $r$ band counts, but extend the study later to other colour bands. It is desirable to work with number counts at a level as faint as possible, so that the galaxy number density is sufficiently large to minimise the Poisson noise and the spatial distribution of galaxies is sufficiently smooth to minimise the large-scale clustering effects. Paper I uses the data in the magnitude range of $r=18.5-20.5$. In the current study, this approach is not appropriate; the reddening expected in the SFD map varies from $E(B-V)=0.03$ to 0.9 (regions of the largest extinction are discarded). This range corresponds to $A_{r}=0.08-2.5$, and the count in highly reddened regions falls out of the range set as the reference magnitude band. To avoid this problem we set the range in a way that dereddened magnitude range is the same for all regions. We use the counts whose number density per square degrees per $0.5 \mathrm{mag}$ is

$$
1.8<\log N(m)<2.6,
$$

and fit $N(m)$ to the reference count to derive $\Delta m$. This range corresponds to $r=17.5-19.5$ on the reference galaxy number count, which is one mag brighter than that in Paper I. This is still reasonably faint, yet photometric measurements are made at a high signal-to-noise ratio and star-galaxy separation is sufficiently reliable even with extinction. This ability to classify objects is particularly important for our study because the contamination of stars becomes more serious at low Galactic latitude where the star density is high and high extinction pushes the objects to fainter magnitudes. The number of galaxies contained in $2.5^{\circ} \times 2.5^{\circ}$ area integrated over $1.8<\log N(m)<2.6$ is approximately 4100 . The expected Poisson noise of $1.6 \%$ is negligible for the present work. It occasionally happens that the faint end of eq. (11) goes beyond the magnitude at which incompleteness starts $(r=22.0)$; in this case we drop the data at the bins that go beyond the incompleteness limit. Figure 4 shows examples of $r$-band galaxy counts in low and high extinction regions. We see in this figure how magnitude offsets are evaluated.

The normalisation of the reference number count in the current sample is lower than 
that derived in Paper I by $5 \%(\Delta \log N(m)=0.02) 2$. A half of this difference is explained by the zero point offset of photometry between DR1 and Finkbeiner et al. (2004), the latter being fainter by $\approx 0.02 \mathrm{mag}$. The other half is due to the present omission of objects that produce this spike at zero surface brightness explained above: we did not apply this cut in Paper I.

We carry out a similar analysis for four other colour bands. The reference range of counts $(\operatorname{as} \log N$ ), the corresponding dereddened magnitude range, approximate number of galaxies, and the limiting magnitude are given in Table 1. Note that we must choose a range of number of galaxies that is smaller in the bluer bands. In particular, the $u$ counts go quickly out of the reference range in the presence of extinction. After deriving reference number counts in the extinction-free limit of the low extinction galaxy samples, we compute $\Delta m_{\lambda}$ for the counts in each group. In deriving the reference counts we assume the standard extinction curve, $k(\lambda)=A_{\lambda} / E(B-V)$ with $k(\lambda)=5.155,3.793,2.086$, and 1.479 for $u, g$, $i$, and $z$. Note that $k(\lambda)$ varies by a factor of 3.5 across $u$ to $z$, and by a factor 2.5 if we drop the $u$ band. This makes the use of $g, r, i, z$ colours appropriate to study the extinction curve, even if we would exclude $u$ for its poorer photometry.

We find the offset in the normalisations of the reference count, $\Delta \log N(m)=0.3,0.03,0.03$, and 0.05 for $u, g, i$, and $z$ compared to those in Paper I. The offsets in $g, i$, and $z$ bands are explained in the same way as for the $r$ band. The offset in $u$ band is large. The shape of the counts at the faint end also differs from that in paper I. These differences originate from the fact that $u$ band surface brightness is faint for many galaxies and the photon collecting efficiency in $u$ band is low, both contributing to poor photometry in this colour band. A comparison of the photometry between DR1 and Finkbeiner et al. (2004) for common galaxies shows that the two magnitudes differ typically as much as 0.5 mag randomly 3 . There are also some differences in the selection procedure. In the present analysis we required 'detection' in the $u$ band in $1 \times 1,2 \times 2$, or $4 \times 4$ binned image, which we did not do in Paper I. The surface brightness cut in $r$ is exercised in the present analysis. The effect is small for the $r$ band, but this drops $10 \%$ of objects for $18.0<u<20.0$ in the $u$ band count. These effects altogether might induce a large error in for the $u$ band count. We expect, however, a substantial part of the errors are likely to cancel when we deal with relative quantities, like those in our analysis.

\footnotetext{
${ }^{2}$ It is lower by $7 \%(\Delta \log N(m)=0.03)$ than Yasuda et al. (2001) which is based on the SDSS Early Data Release (Stoughton et al. 2002).

${ }^{3}$ For stars this large scatter is not seen. The difference of the two magnitudes is no more than twice those in $r$ or $g$ bands; the mean scatter is 0.03 mag at $u=19$.
} 


\section{Results}

In Figure 5 we show $\Delta m_{r}$ versus Galactic extinction, $A_{r}^{\mathrm{SFD}}$, calculated from the reddening map of SFD averaged over each group with the effective area of $\left(2.5^{\circ}\right)^{2}$ assuming the standard extinction curve. For each group, we applied a jackknife method with the data divided into 10 samples to estimate statistical errors. The horizontal bars stand for the range of $A_{r}$ in each group. The data points for which the faint end of the observed magnitude corresponding to eq. (1) becomes fainter than the incompleteness limit are shown by open circles. We note that the scatter of the points is substantially reduced compared to those in Paper I, where it is dominated by large scale clustering of galaxies. We expect a \pm 0.11 mag variation from large scale galaxy clustering if the area considered were taken from contiguous regions along the stripe. The observed scatter $(0.04 \mathrm{mag})$ is consistent with the mean error of fitting of the reference count to the data of $N(m)$, which is 0.04 . This reduced scatter obviously arises from the fact that the information of the large scale structure is mostly lost by division and reassembling of the area. This is an advantage of the present procedure from the viewpoint of testing the effect of extinction.

Figure 5 shows that for $A_{r}^{\mathrm{SFD}}<0.4$ (or $\left.E(B-V)<0.15\right), \Delta m_{r}$ is proportional to $A_{r}^{\mathrm{SFD}}$ when averaged over the scatter. This agrees with what we demonstrated in Paper I: the galaxy counts are consistent with the reddening map and the standard extinction law. For a high extinction regime, $A_{r}^{\mathrm{SFD}}>0.4$ or $E(B-V)>0.15$, however, the data significantly deviate from the identity regression line: $\Delta m_{r}$ is smaller than $A_{r}^{\mathrm{SFD}}$, indicating that $A_{r}^{\mathrm{SFD}}$ overestimates the Galactic extinction.

This overestimate of Galactic extinction in the SFD map is consistent with the analysis of Arce \& Goodman (1999a, b) , who studied extincion in the Taurus dark cloud complex using the number count and reddening of background stars and $100 \mu \mathrm{m}$ infrared emission (calibrated with star number counts). They reached the conclusion that the SFD map overestimates the extinction by a factor of $1.3-1.5$ for the region of $A_{V}>0.5$.

We perform a similar analysis for the $u, g, i$, and $z$ colour bands. The relations between $\Delta m_{\lambda}$ and $A_{\lambda}^{\mathrm{SFD}}$ are shown in Figure 6. For the $g, i$ and $z$ bands we see a similar departure from the $\Delta m_{\lambda}=A_{\lambda}^{\mathrm{SFD}}$ line for $E(B-V) \gtrsim 0.15$, while the data for $E(B-V) \lesssim 0.15$ are consistent with the identity line. The departure for the $u$ band appears somewhat different, but we note that the data for $A_{u}^{\mathrm{SFD}}>2$, where a significant departure is observed, are derived from the counts whose faint end extends beyond the incompleteness limit, in addition to the problem of photometry discussed above. We take the results with the $u$ band hereinafter only for the purpose to see the broad, rather than quantitative, consistency.

The departure from the identity regression is more clearly demonstrated in Figure 7. 
where the ratio of $\Delta m_{\lambda} / A_{\lambda}^{\mathrm{SFD}}$ is plotted as a function of $A_{\lambda}^{\mathrm{SFD}}$ for each band. The mean values are presented as horizontal bars (for numerical values see Table 2) for the ranges of $E(B-V)=0.05-0.15,0.15-0.45$, and $0.40-1.00$. For $0.05<E(B-V)<0.15$, these ratios are consistent with unity within $1 \sigma$ errors. Apparent departure from unity, $\Delta m_{\lambda}<A_{\lambda}^{\mathrm{SFD}}$, is visible for higher extinction ranges. The ratio $A_{\lambda}^{\mathrm{SFD}} / \Delta m_{\lambda}$ is approximately 1.25 for $E(B-V)=0.15-0.45$, and 1.4 for $E(B-V)=0.45-1.00$ irrespective of the colour bands (again except for the $u$ band).

Two alternative interpretations for this observed departure are (1) the reddening function $k(\lambda)$ is non-linear for a large $A_{\lambda}$ or $(2)$ the selective reddening $E(B-V)$ estimated by SFD is not correct for large extinction. To distinguish between the two possibilities, the regressions of $\Delta m_{\lambda}$ among different colour bands are plotted in Figure 8. The dotted lines show the relations expected for the standard extinction law, i.e., $\Delta m_{\lambda_{1}} / \Delta m_{\lambda_{2}}=k\left(\lambda_{1}\right) / k\left(\lambda_{2}\right)$. Note that the scales of the abscissa and ordinate are not identical. The figure shows that the data points follow the expected relation; no non-linearity is observed in the extinction curve across the regions of low to high extinction.

We may estimate $k(\lambda)$ relative to the reference band, which we take to be the $r$ band. Defining $k_{\lambda}^{\prime}=k(\lambda) / k(r)$, we can calculate this quantity from the observed relation $\Delta m_{\lambda}=$ $k_{\lambda}^{\prime} \cdot \Delta m_{r}$. The results from this analysis are shown in Figure 9. The $k$ values in Table 3 are obtained by multiplying $k(r)=2.751$ on $k_{\lambda}^{\prime}$. The observed values of $k(\lambda)$ are consistent with those of the standard extinction curve.

This analysis suggests that $E(B-V)$ of SFD is overestimated for high extinction regions. Using $\Delta m_{\lambda} / A_{\lambda}^{\mathrm{SFD}}$ from $g$ to $z$ bands, we suggest the true selective reddening being written as

$$
E(B-V)^{\text {true }}=E(B-V)^{\mathrm{SFD}}\left[0.87-0.13 \operatorname{Erf}\left(\frac{E(B-V)^{\mathrm{SFD}}-0.19}{0.11}\right)\right]
$$

in terms of $E(B-V)^{\mathrm{SFD}}$. The prediction of this function is presented in Figure 7 ,

In agreement with the analysis of Arce \& Goodman (1999a) we ascribe the overestimate of $E(B-V)$ by SFD to the inaccuracy of the conversion of $100 \mu m$ emission of dust $\left(D^{T}\right)$ to selective reddening for high extinction regions. SFD assume a simple linear relation between reddening and $100 \mu m$ emission as $E(B-V)=p D^{T}$, where $p$ is a parameter. To determine $p$, SFD use the relation between the intrinsic $B-V$ colour and the $\mathrm{Mg}_{2}$ line index of elliptical galaxies. Of the 389 elliptical galaxies used, only $\approx 20$ reside in regions with $E(B-V)>0.15$, and there are no elliptical galaxies in the region of $E(B-V)>0.4$. We notice that the fitting of $100 \mu m$ vs $E(B-V)$ relation of SFD (see their Figure 6) starts deviating from linear relation for $E(B-V) \gtrsim 0.15$. From their Figure 6 , the value of $\delta(B-V)$ is about $-0.08 \mathrm{mag}$ for elliptical galaxies whose $E(B-V)$ s are between 0.2 and 0.4 ; this 
corresponds to overestimation of reddening by $1.2-1.4$ when a linear relation is assumed. This is quantitatively consistent with our result. The relation between the reddening and $100 \mu \mathrm{m}$ emission should be modified to incorporate the non-linearity.

We briefly discuss the possible reasons for the departure between $100 \mu \mathrm{m}$ emission and reddening from a linear relation. Overestimation of reddening can be caused by the overestimation of $100 \mu \mathrm{m}$ emission. According to the procedure of SFD, $100 \mu \mathrm{m}$ emission will be overestimated when color temperature estimated from the ratio of intensities at 100 and 240 $\mu m$ is underestimated. If dense clouds have larger dust grains, their equilibrium temperature will be lower even in the same radiation field. This will cause overestimation of $100 \mu m$ emission. Could the composition be different? SFD assumed the emissivity model of $\epsilon_{\nu}=\nu^{\alpha}$ with $\alpha=2.0$. If there are materials with $\alpha=1.5$, their temperature will be underestimated. This is the same sense as we have seen. How about mixed temperature along the line of sight? From Figure 2 of SFD the column density will be underestimated when there are two regions at different equilibrium temperatures. This is the opposite sense to what we have seen. From these brief discussion, the difference of grain size and emissivity model in dense clouds could be the cause of overestimation of $100 \mu \mathrm{m}$ emission. However, we need more detailed observations to explore the ways of modifying the SFD map.

\section{Summary}

We have tested Galactic extinction using galaxy number counts at the low Galactic latitude using the SDSS galaxy sample of Finkbeiner et al. (2004) that covers $200 \mathrm{deg}^{2}$ from low $E(B-V) \approx 0.03$ to high $E(B-V) \approx 1$ extinction regions. The variation of galaxy number counts is consistent with Galactic extinction described by the prediction of SFD for low extinction regions of $E(B-V)<0.15$. For high extinction regions of $E(B-V)>0.15$, the SFD extinction prescription overestimates the reddening by a factor up to 1.4 , which we

interpret as a result of the departure of the linear relation between $100 \mu \mathrm{m}$ infrared emission and selective extinction.

We thank Jill Knapp for invaluable suggestions for improving the analysis.

Funding for the SDSS and SDSS-II has been provided by the Alfred P. Sloan Foundation, the Participating Institutions, the National Science Foundation, the U.S. Department of Energy, the National Aeronautics and Space Administration, the Japanese Monbukagakusho, the Max Planck Society, and the Higher Education Funding Council for England. The SDSS Web Site is http://www.sdss.org/. 
The SDSS is managed by the Astrophysical Research Consortium for the Participating Institutions. The Participating Institutions are the American Museum of Natural History, Astrophysical Institute Potsdam, University of Basel, Cambridge University, Case Western Reserve University, University of Chicago, Drexel University, Fermilab, the Institute for Advanced Study, the Japan Participation Group, Johns Hopkins University, the Joint Institute for Nuclear Astrophysics, the Kavli Institute for Particle Astrophysics and Cosmology, the Korean Scientist Group, the Chinese Academy of Sciences (LAMOST), Los Alamos National Laboratory, the Max-Planck-Institute for Astronomy (MPIA), the Max-Planck-Institute for Astrophysics (MPA), New Mexico State University, Ohio State University, University of Pittsburgh, University of Portsmouth, Princeton University, the United States Naval Observatory, and the University of Washington.

\section{REFERENCES}

Abazajian, K. et al. 2003, AJ, 126, 2081

Abazajian, K. et al. 2004, AJ, 128, 502

Abazajian, K. et al. 2005, AJ, 129, 1755

Adelman-McCarthy, J. K. et al. 2006, ApJS, 162, 38

Adelman-McCarthy, J. K. et al. 2007, ApJS, in press

Arce, H. G., \& Goodman, A. A. 1999, ApJ, 512, L135

Arce, H. G., \& Goodman, A. A. 1999, ApJ, 517, 264

Blanton, M. R. et al. 2001, AJ, 121, 2358

Burstein, D., \& Heiles, C. 1982, AJ, 87, 1165

Cardelli, J. A., Clayton, G. C., \& Mathis, J. S. 1989, ApJ, 345, 245

Finkbeiner, D. P. et al. 2004, AJ, 128, 2577

Fukugita, M., Ichikawa, T., Gunn, J. E., Doi, M., Shimasaku, K., \& Schneider, D. P. 1996, AJ, 111,1748

Fukugita, M., Yasuda, N., Brinkmann, J., Gunn, J. E., Ivezić, Ž., Knapp, G. R., Lupton, R., \& Schneider, D. P. 2004, AJ, 127, 3155 (PaperI) 
Gunn, J. E. et al. 1998, AJ, 116, 3040

Gunn, J. E. et al. 2006, AJ, 131, 2332

Hogg, D. W., Finkbeiner, D. P., Schlegel, D. J., \& Gunn, J. E. 2001, AJ, 122, 2129

Hubble, E. 1934, ApJ, 79, 8

Ivezić, Ž. et al. 2004, AN, 325, 583

O’Donnell, J. E. 1994, ApJ, 422, 158

Pier, J. R. 2003, AJ, 125, 1559

Schlegel, D. J., Finkbeiner, D. P., \& Davis, M. 1998, ApJ, 500, 525 (SFD)

Smith, J. A. et al. 2002, AJ, 123, 2121

Stoughton, C. et al. 2002, AJ, 123, 485

Strauss, M. A. et al. 2002, AJ, 124, 1810

Tucker, D. et al. 2006, Astron. Nachr., 327, 821

Yahata, K. et al. 2006, astro-ph/0607098 (sumitted to PASJ)

Yasuda, N. et al. 2001, AJ, 122, 1104

York, D. G. et al. 2000, AJ, 120, 1579 
Table 1. Parameters for our analysis

\begin{tabular}{ccccc}
\hline \hline Band & $\begin{array}{c}\text { Range of counts } \\
\log N(m)\end{array}$ & Mag range & Num. of gal & Limiting mag \\
\hline$u$ & $1.0-1.8$ & $18.0-20.0$ & 800 & \\
$g$ & $1.3-2.1$ & $17.5-19.5$ & 1600 & 21.6 \\
$r$ & $1.8-2.6$ & $17.5-19.5$ & 4100 & 22.4 \\
$i$ & $1.8-2.6$ & $17.0-19.0$ & 3800 & 21.2 \\
$z$ & $1.8-2.6$ & $16.5-18.5$ & 3100 & 19.8 \\
\hline
\end{tabular}

Table 2. Mean values of $\Delta m_{\lambda} / A_{\lambda}^{\mathrm{SFD}}$

\begin{tabular}{cccc}
\hline \hline & \multicolumn{3}{c}{ Range of $E(B-V)$} \\
\cline { 2 - 4 } Band & $0.05-0.15$ & $0.15-0.45$ & $0.45-1.00$ \\
\hline$u$ & $1.130 \pm 0.063$ & $0.950 \pm 0.019$ & $\ldots$ \\
$g$ & $1.095 \pm 0.085$ & $0.829 \pm 0.015$ & $0.718 \pm 0.031$ \\
$r$ & $1.017 \pm 0.055$ & $0.818 \pm 0.022$ & $0.734 \pm 0.019$ \\
$i$ & $1.028 \pm 0.075$ & $0.791 \pm 0.038$ & $0.694 \pm 0.023$ \\
$z$ & $1.079 \pm 0.103$ & $0.800 \pm 0.057$ & $0.681 \pm 0.031$ \\
\hline
\end{tabular}

Table 3. The value of $k(\lambda)$

\begin{tabular}{ccl}
\hline \hline Colour band & Standard extinction curve & Derived from $\Delta N(m)$ \\
\hline$u$ & 5.155 & $5.611 \pm 0.165$ \\
$g$ & 3.793 & $3.779 \pm 0.045$ \\
$r$ & 2.751 & 2.751 (normalisation) \\
$i$ & 2.086 & $1.9748 \pm 0.015$ \\
$z$ & 1.479 & $1.397 \pm 0.019$ \\
\hline
\end{tabular}




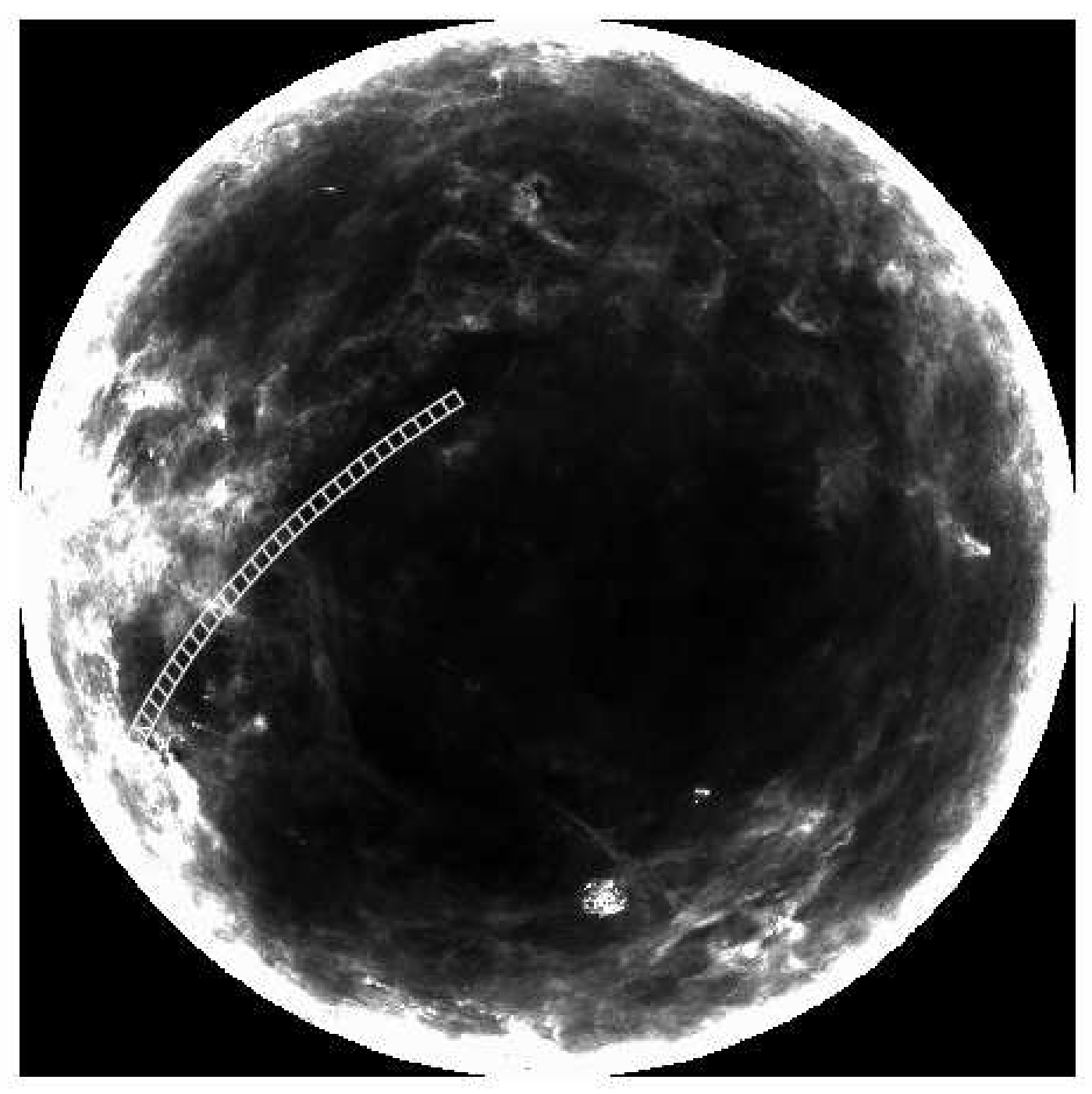

Fig. 1. - Region for the current analysis overlaid on the reddening map of SFD in the Galactic southern hemisphere with the pole at the centre $\left(b=-90^{\circ}\right)$. The left edge corresponds to $l=180^{\circ}$ and the top is $l=90^{\circ}$. The stripe runs from $(l, b)=\left(125^{\circ},-62^{\circ}\right)$ to $\left(207^{\circ},-10^{\circ}\right)$ and the square along the stripe represents $2.5^{\circ}$ square regions. The progression of black to white in the figure corresponds to increasing values of $E(B-V)$. 


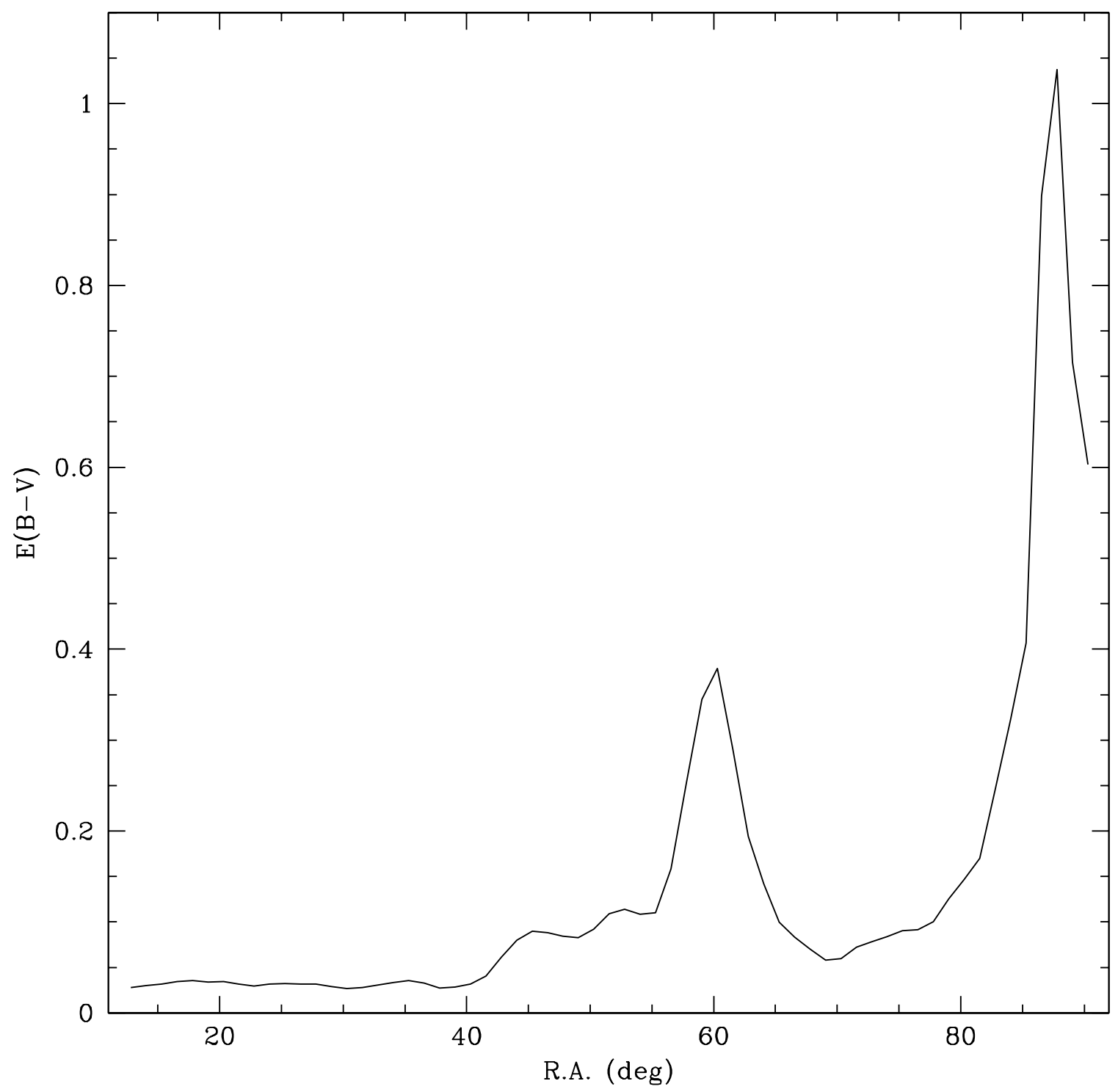

Fig. 2.- Variation of the mean reddening $E(B-V)$ of SFD averaged over the $2.5^{\circ}$ square region along the stripe in this study. The Orion Complex is located at R.A. $=90^{\circ}$. 


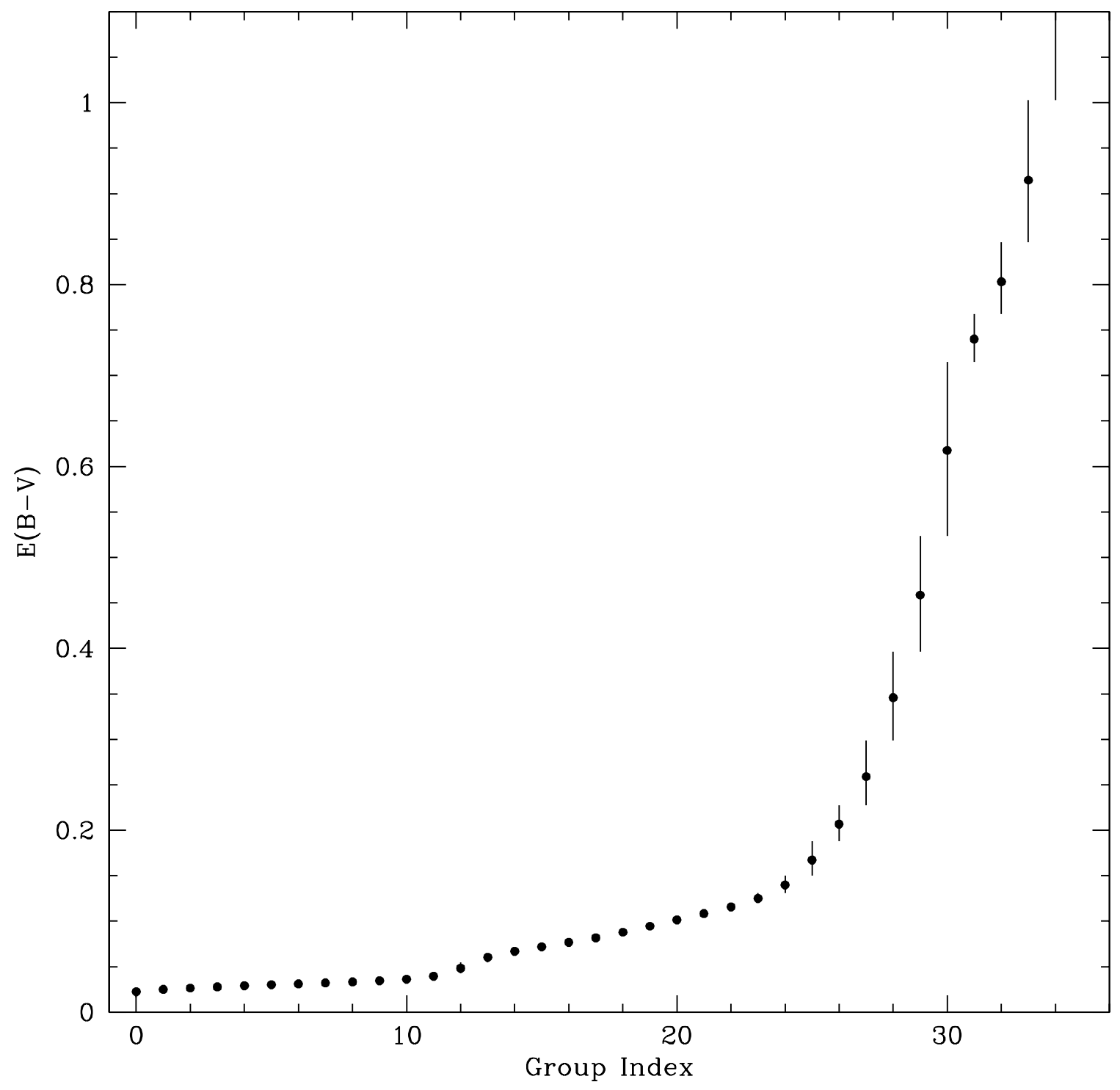

Fig. 3.- Range of $E(B-V)$ of the SFD map for 32 groups, each group consisting of 4225 pixels that form the effective $2.5^{\circ}$ square region. (The four rightmost points are the groups having one fourth the number of pixels of the others, corresponding to effective $1.25^{\circ}$ square.) Filled circles denote the mean values and bars show the minimum and maximum. 


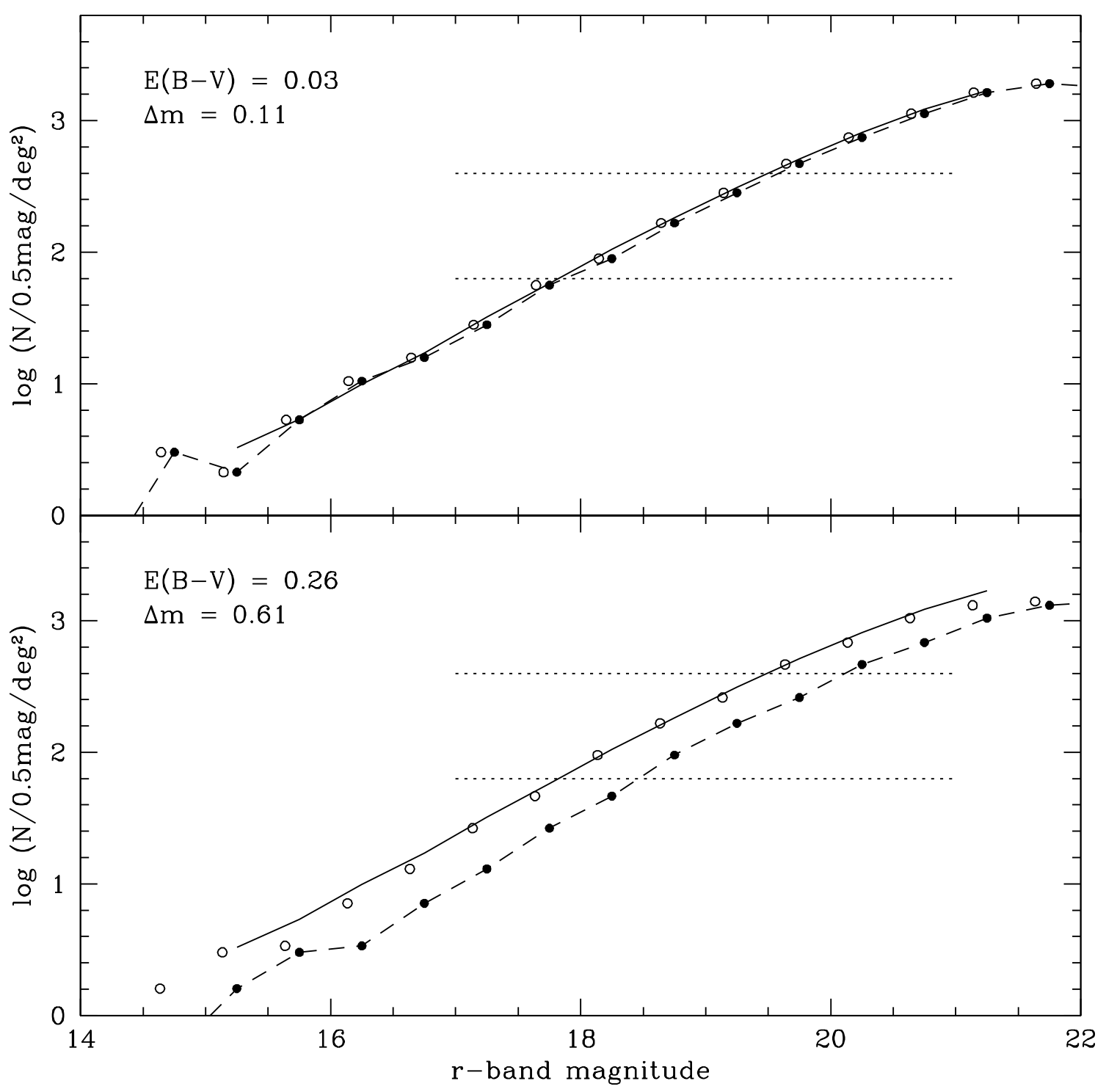

Fig. 4.- Example galaxy number counts in the $r$-band. Upper panel is for a low extinction $(E(B-V)=0.03)$ region and lower panel is for a high extinction $(E(B-V)=0.26)$ region. Solid lines are the reference galaxy counts. Solid points (and dashed lines) represent observed galaxy counts, which are shifted to match the reference counts by the amount of $\Delta m$ (denoted by open circles). The bands indicated by dotted lines are the range that is used for matching. 


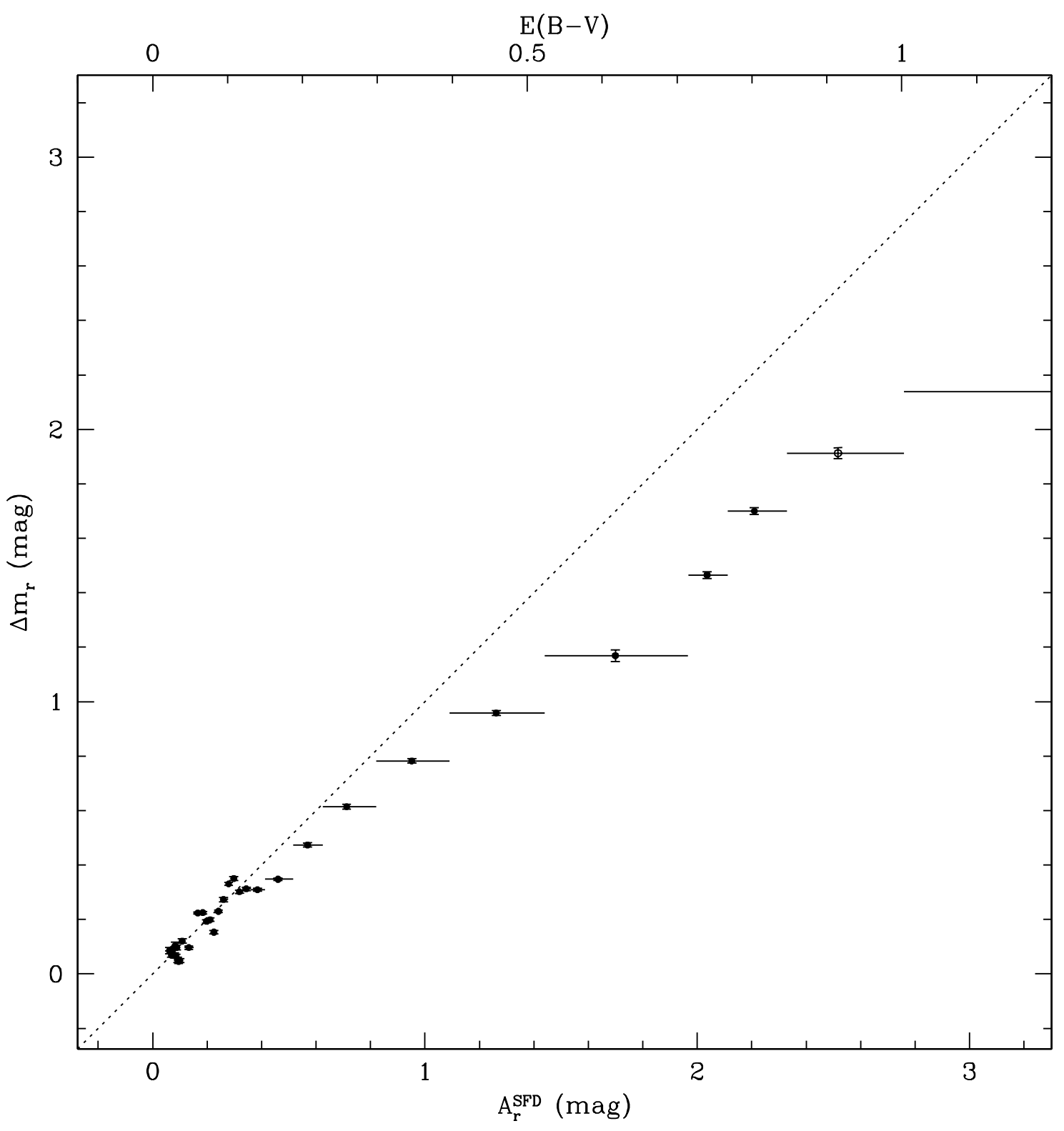

Fig. 5.- Magnitude offsets $\Delta m_{r}$ corresponding to the variation of galaxy number counts in groups corresponding to the effective area of $\left(2.5^{\circ}\right)^{2}$ plotted against mean extinction $A_{r}^{\mathrm{SFD}}$ calculated from the SFD reddening map and the standard extinction curve. Horizontal bars show the region of $A_{r}$ in each group and vertical bars are errors obtained by a jackknife estimate. The open circles represent the region where $A_{r}^{\mathrm{SFD}}$ is so large that the faint end of the reference magnitude range falls beyond the incompleteness limit. The dotted line is the identity regressions line $\Delta m_{r}=A_{r}^{\mathrm{SFD}}$. 

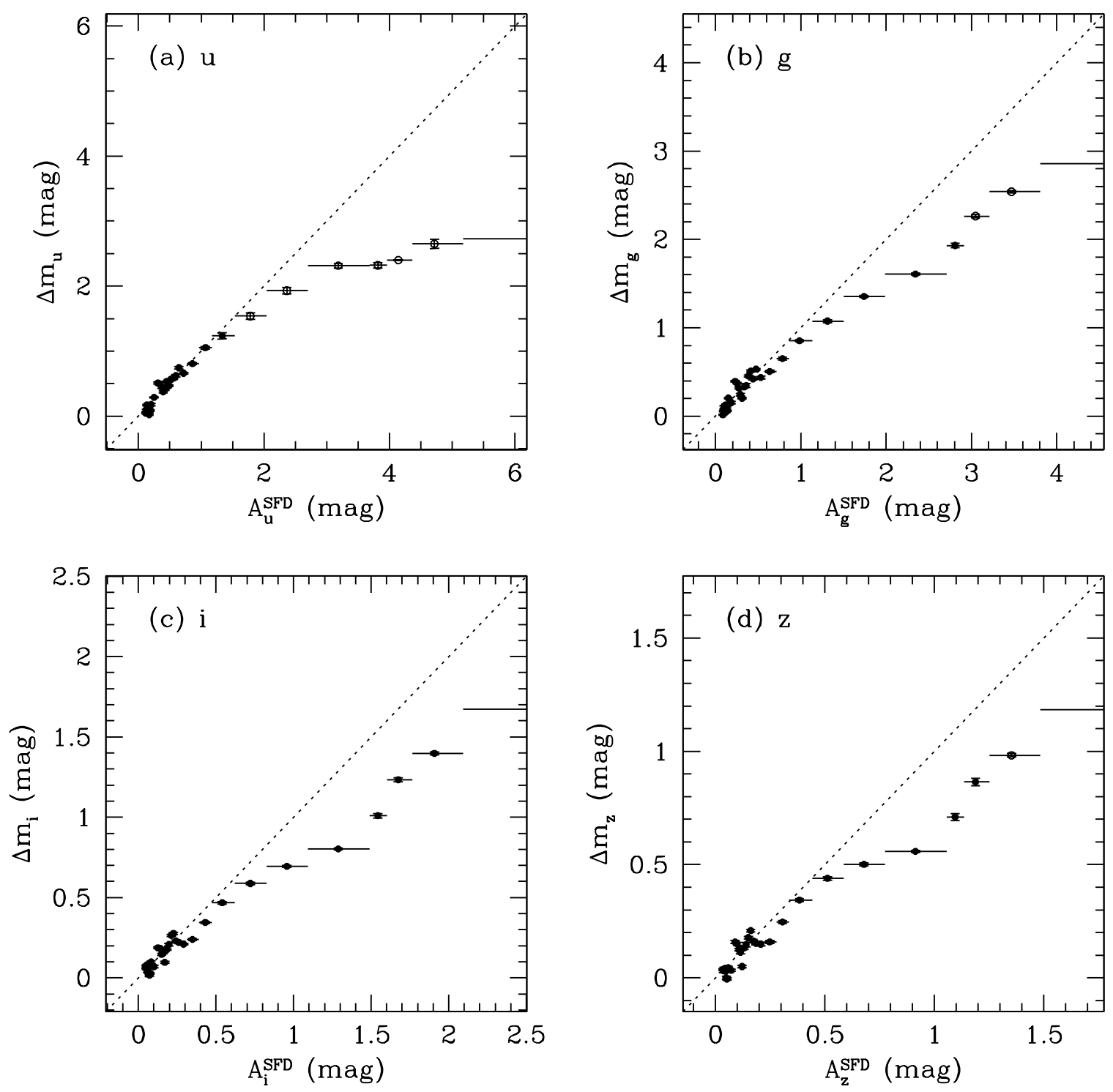

Fig. 6. - Same as Figure 5, but for the other four SDSS colour bands: (a) $u$ band, (b) $g$ band, (c) $i$ band, and (d) $z$ band. 

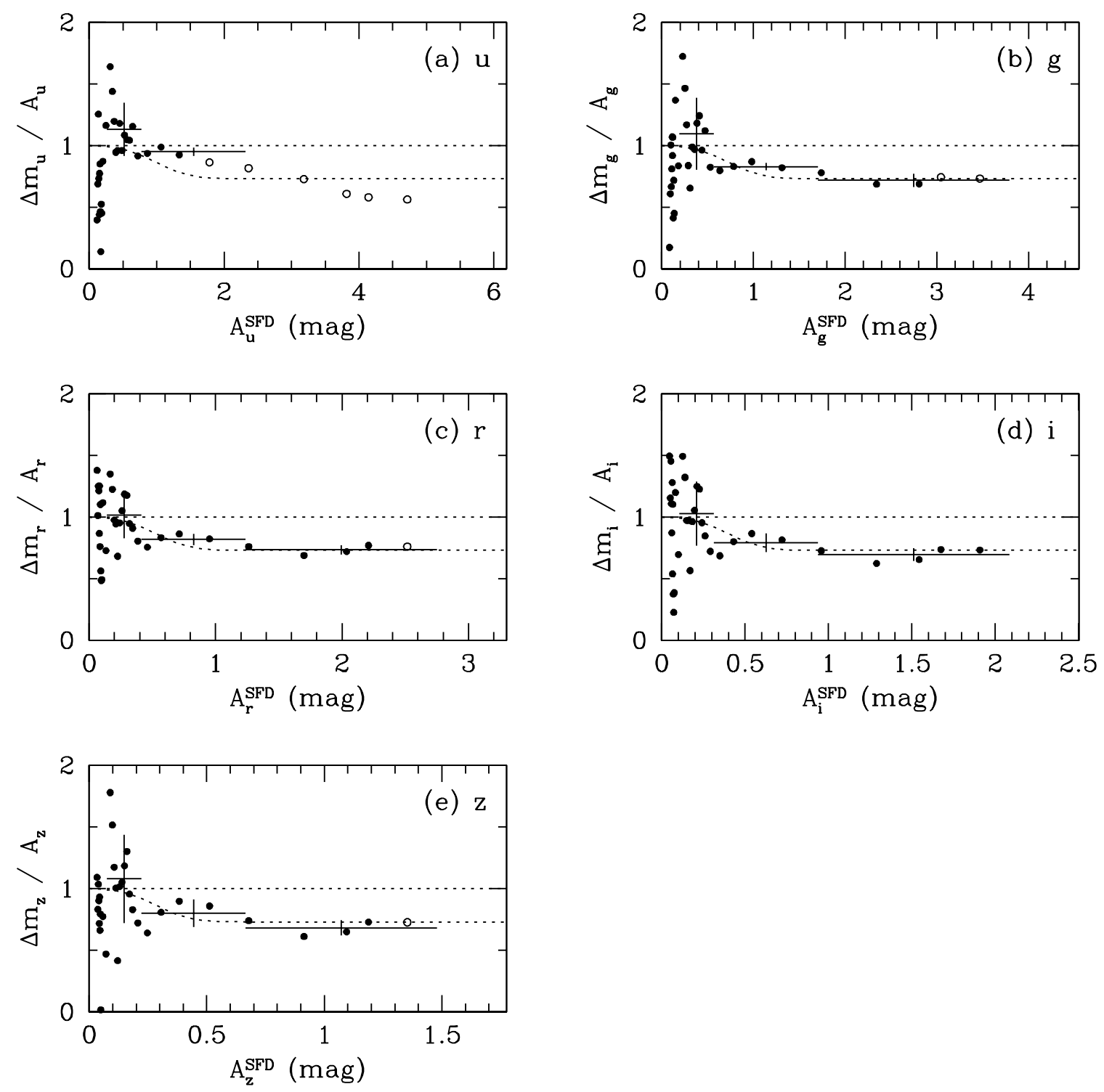

Fig. 7.- The ratio of $\Delta m_{\lambda} / A_{\lambda}^{\mathrm{SFD}}$ as a function of $A_{\lambda}^{\mathrm{SFD}}$ for five colour bands. The meaning of open circles is the same as in Figure 5. The mean values for different ranges of $E(B-V)$ $(0.05-0.15,0.15-0.45$, and $0.45-1.00)$ are indicated as horizontal bars, and the empirical fitting function eq. (2) is shown with dotted curves. 


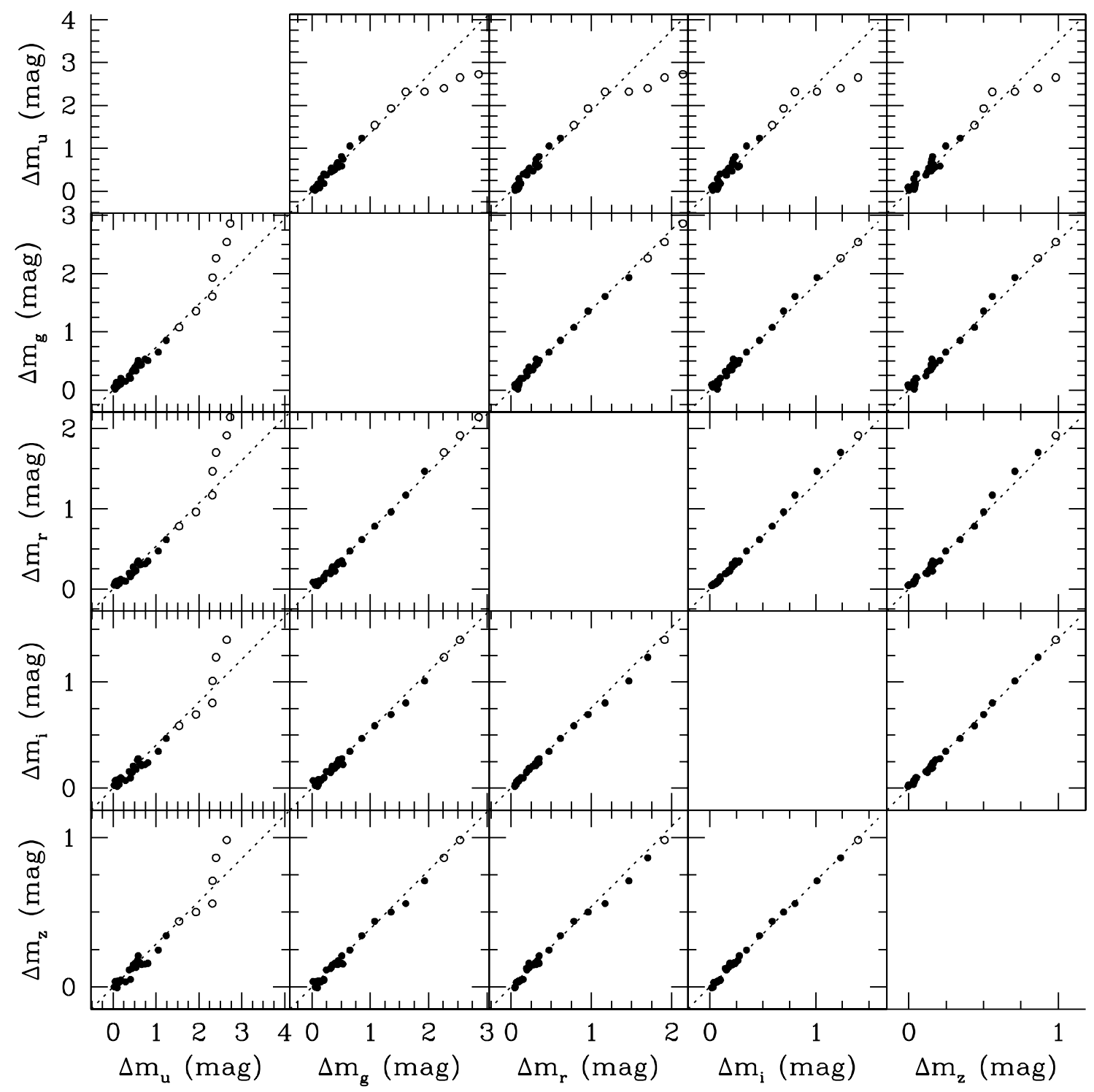

Fig. 8.- The regression of $\Delta m_{\lambda}$ between different combinations of $u, g, r, i$, and $z$. The meaning of open circles is the same as in Figure 5. The dotted lines are relations expected for the standard extinction law. 


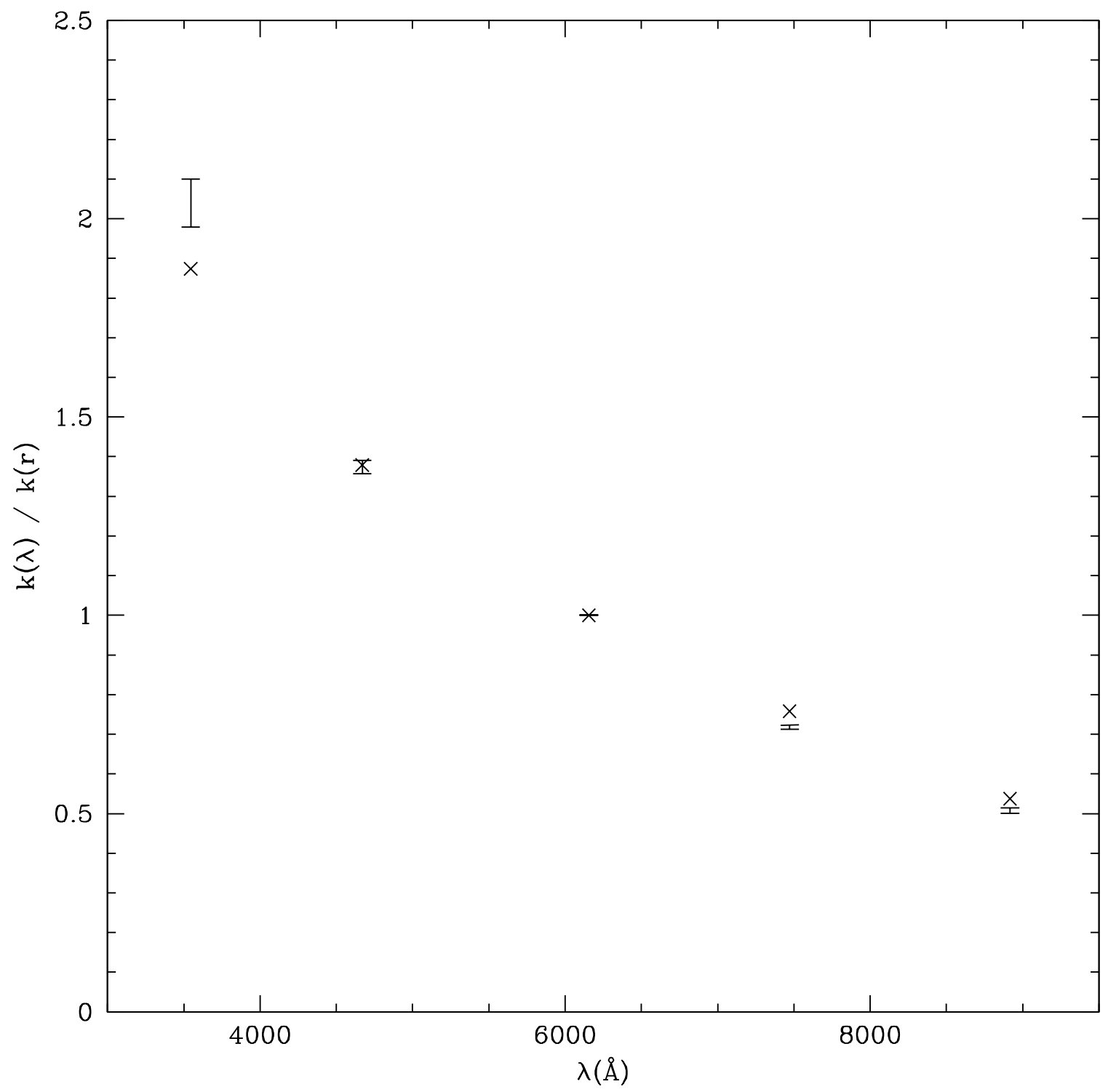

Fig. 9.- $k(\lambda) / k(r)$ obtained from galaxy number counts as $\Delta m_{\lambda} / \Delta m_{r}$ (data with error bars) and those from the standard extinction curve (crosses). 